\title{
Data assimilation of post irradiation examination experiments to adjust fission yields
}

\author{
Daniel Siefman ${ }^{1, *}$, Mathieu Hursin ${ }^{1,2}$, and Andreas Pautz ${ }^{1,2}$ \\ ${ }^{1}$ Institute of Physics, LRS, École Polytechnique Fédérale de Lausanne (EPFL), 1025 Lausanne, Switzerland \\ ${ }^{2}$ Nuclear Energy and Safety, Paul Scherrer Institut (PSI), 5232 Villigen, Switzerland
}

\begin{abstract}
Nuclear data, especially fission yields, create uncertainties in the predicted concentrations of fission products in spent fuel. Herein, we present a new framework that extends data assimilation methods to burnup simulations by using data from post-irradiation examination experiments. The adjusted fission yields improve the bias and reduce the uncertainty of predicted fission product concentrations in spent fuel. Our approach modifies fission yields by adjusting the model parameters of the code GEF with post-irradiation examination experiments. We used the BFMC data assimilation method to account for the non-normality of GEF's fission yields. In the application that we present, the assimilation decreased the average bias of the predicted fission product concentrations from $26 \%$ to $15 \%$. The average relative standard deviation decreased from $21 \%$ to 14\%. The GEF fission yields after data assimilation agreed better with those in ENDF/B-VIII.0. For Pu-239 thermal fission, the average relative difference from ENDF/B-VIII.0 was $16 \%$ before data assimilation and $11 \%$ after. For the standard deviations of the fission yields, GEF's were, on average, $16 \%$ larger than those from ENDF/B-VIII.0 before data assimilation and $15 \%$ smaller after.
\end{abstract}

\section{Introduction}

Fission product yields (FYs) and their uncertainties are very important for burn-up [1-3], decay heat [4, 5], and nuclear waste management calculations [6]. These simulations are fundamentally based on predicting the concentration of fission products (FPs) in spent fuel, which requires reliable FY data with high quality covariances. Historically, nuclear data libraries did not consistently and completely provide uncertainty information about FYs [7]. In many cases, only nominal values and variances were given; no covariances were provided.

One way to produce FY uncertainty distributions is with the code GEF [8-10]. Through Monte Carlo sampling of its model parameters, GEF outputs sample sets of FYs that can be used for uncertainty quantification in burnup. Unfortunately, these FYs create uncertainties in burnup simulations. For the fuel in this study, the uncertainties are as large as $56 \%$, with an average of $21 \%$. Because of this, GEF's FYs are an interesting target for data assimilation (DA), i.e. the adjustment of nuclear data with integral experiments [11].

In this study, we do DA with experimental postirradiation examination (PIE) data. PIE data have recently been used to adjust cross sections [12,13], but never with FYs and only with sensitivity-based approaches [14]. Through the incorporation of experimental evidence, we adjust the model parameters of GEF and subsequently the FYs it calculates. The goal is that these adjusted FYs lead

*e-mail: daniel.siefman@epfl.ch

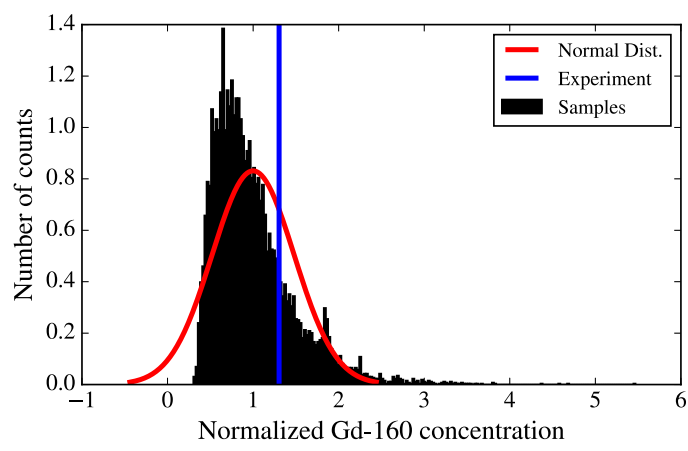

Figure 1. Histogram of the prior calculated Gd-160 concentrations normalized to the mean.

to smaller biases and reduce uncertainties of simulated FP concentrations in spent fuel.

PIE data present particular challenges for DA because GEF's FYs can be non-Gaussian. When they are used in calculations, they create non-Gaussian distributions in the calculated FP concentrations (Fig. 1). The non-Gaussianity makes the assumptions in other DA methods like GLLS [15] or MOCABA [16] inaccurate, thereby limiting their effectiveness. In this work, we used the Backward-Forward Monte Carlo (BFMC) [17] DA method to account for the non-normality. We adapted work presented in Ref. [18] that used ENDF/B-VII.1 [19] FY data as the experimental evidence in the DA. The fact that GEF inherently does stochastic sampling also allows it 
to be easily integrated into stochastic-sampling-based DA methods [20, 21], such as BFMC.

Another challenge of the data set was its large degree of inconsistency, or its large prior $\chi^{2}$. The inconsistency was in part caused by the approximated irradiation history and geometry in the burnup simulations. It was also caused by experimental bias and unaccounted uncertainties or correlations. The inconsistency may lead to an overfit of the GEF model to the experimental data. To account for this, we used a technique called Marginal Likelihood Optimization (MLO) [22-24]. It accounts for the discrepancy between experimental and calculated integral data by adding extra uncertainty terms that limit the influence of the data on the adjustment. It also compensates for unaccounted sources of uncertainty, like omitting the energy dependence of fission from the fission yield uncertainties.

Herein, we present the adjustments of GEF's model parameters, and subsequently the FY data generated with them, using PIE data from the LWR-Proteus Phase II experiment [25-27]. The goal was to reduce the bias and uncertainty of these predicted FP concentrations. The prior and posterior GEF FYs are also compared to those given in ENDF/B-VIII.0 [28]. Additionally, we demonstrate how DA changes the correlations between FYs.

\subsection{BFMC}

DA for PIE data and GEF can be formulated with Bayes' theory. The prior is GEF's model parameters which are represented by a vector, $\sigma=\left\{\sigma_{i} \mid i=1, \cdots, N_{\sigma}\right\}$, where $N_{\sigma}$ is the number of parameters. Experimental PIE data, $\mathbf{E}$, are used to update the prior and are represented as the vector, $\mathbf{E}=\left\{E_{i} \mid i=1, \cdots, N_{E}\right\}$, where $N_{E}$ is the number of experimental parameters. The prior and the data together lead to the likelihood function, $\mathcal{L}(\mathbf{E} \mid \mathbf{C}(\sigma))$. Here, $\mathbf{C}(\sigma)$ is a vector of simulated PIE data using the FYs created by GEF with the model parameters $\sigma$. Finally, the posterior is $p\left(\sigma^{\prime} \mid \mathbf{E}\right)$, given as Bayes' theorem in Eq. (1).

$$
p\left(\sigma^{\prime} \mid \mathbf{E}\right) \propto \mathcal{L}(\mathbf{E} \mid \mathbf{C}(\sigma)) p(\sigma)
$$

Backward Forward Monte Carlo (BFMC) is a DA method based on stochastic sampling. It stochastically seeks to maximize the agreement between $\mathbf{C}(\boldsymbol{\sigma}))$ and $\mathbf{E}$, within the constraint of the prior, to calculate $p\left(\sigma^{\prime} \mid \mathbf{E}\right)$. It makes no assumptions about the distribution of the prior or likelihood [29]. The process begins by randomly sampling the nuclear data from their prior distribution. For every randomly-sampled model parameter set, $\sigma_{i}$, GEF calculates FYs which are then used in a simulation of the PIE data, $\mathbf{C}\left(\sigma_{i}\right)$, or $\mathbf{C}_{i}$. When $N$ samples of $\boldsymbol{\sigma}$ are performed, the PIE data are simulated $N$ times to create a population of calculated values: $\mathbf{C}_{1}, \mathbf{C}_{2}, \ldots, \mathbf{C}_{N}$. After random sampling, each $\mathbf{C}_{i}$ is compared to $\mathbf{E}$ to find how it changed the likelihood. The likelihood for $i^{\text {th }}$ sample is given in Eq. (2), where $\chi_{i}^{2}$ is calculated with Eq. (3).

$$
\begin{gathered}
\mathcal{L}\left(\mathbf{E} \mid \mathbf{C}_{i}\right) \propto \exp \left(\chi_{i}^{2} / 2\right) \\
\chi_{i}^{2}=\left(\mathbf{E}-\mathbf{C}_{i}\right)^{T}\left(\mathbf{M}_{\mathbf{E}}\right)^{-1}\left(\mathbf{E}-\mathbf{C}_{i}\right)
\end{gathered}
$$

Each $\chi_{i}^{2}$ is used to calculate a weight, $w_{i}$, for that $\sigma_{i}$. Smaller $\chi_{i}^{2}$ values, or better agreement between $\mathbf{E}$ and $\mathbf{C}_{i}$, are weighted more heavily. Samples with higher $w_{i}$ contribute more to adjustments, whereas smaller $w_{i}$ contribute less. BFMC calculates the weights with Eq. (4), where $\chi_{i}^{2}$ is normalized with the minimum $\chi^{2}$ of the sample set, or $\chi_{\min }^{2}$. The normalization improves the weight distribution and the convergence rate of the posteriors [30].

$$
w_{i}=\exp \left(\chi_{i}^{2} / \chi_{\min }^{2}\right)
$$

The weights are then used to calculate the posterior means of the model parameters $\sigma^{\prime}$ in Eq. (5), and their posterior covariance matrix $\mathbf{M}_{\sigma}^{\prime}$ in Eq. (6). Here, $\overline{\boldsymbol{\sigma}}$ is the population mean.

$$
\begin{gathered}
\boldsymbol{\sigma}^{\prime}=\frac{\sum_{i=1}^{N} w_{i} \times \boldsymbol{\sigma}_{i}}{\sum_{i=1}^{N} w_{i}} \\
\mathbf{M}_{\sigma}^{\prime}=\frac{\sum_{i=1}^{N} w_{i} \times\left(\boldsymbol{\sigma}_{i}-\overline{\boldsymbol{\sigma}}\right)\left(\boldsymbol{\sigma}_{i}-\overline{\boldsymbol{\sigma}}\right)^{T}}{\sum_{i=1}^{N} w_{i}}
\end{gathered}
$$

\subsection{LWR Proteus Phase II}

The PIE data come from Phase II of the LWR-Proteus campaign. We used the PIE data of one $\mathrm{UO}_{2}$ fuel rod, $\mathrm{U} 1$, which had a burnup of $\sim 38 \mathrm{MWd} / \mathrm{kg}$. The integral data are FP concentrations relative to the total uranium mass in the fuel. In total, 33 integral data points were used. They have a degree of correlation induced by the normalization by the total uranium concentration. The nuclides of an element are also inter-correlated by the use of a reference sample during liquid chromatography and mass spectrometry, creating a common source of uncertainty. These correlations were calculated and put into the matrix $\mathbf{M}_{\mathbf{E}}$ of Eq. (3).

The posterior FYs were also tested on the PIE data of another $\mathrm{UO}_{2}$ fuel rod, U2, which had a burnup of $\sim 58$ $\mathrm{MWd} / \mathrm{kg}$. U2 tested the performance of the posterior FYs for a system that was not included in the adjustment. It assessed how the adjusted FYs generalize to other systems, i.e. if they were overfit to $\mathrm{U} 1$.

\subsection{Approach}

We used CASMO-5M [31] to model the LWR-PII fuel samples and to predict the concentrations of FPs after burnup. The CASMO-5M models are described in Ref. [26]. The tool SHARK-X [32-35] transferred the FY samples from GEF2017/1.2 to CASMO-5M. GEF created FYs and covariance data for the thermal fission (incident neutron energy of $0.0253 \mathrm{eV}$ ) of $\mathrm{U}-235, \mathrm{Pu}-239$, and $\mathrm{Pu}-241$, and the fast fission (incident neutron energy of $500 \mathrm{keV}$ ) of U-238, all with 2E5 fission events.

GEF's model parameters were sampled 10,000 times and GEF created 10,000 sets of FYs. Here, the model parameters were sampled from independent, normal distributions. For each sample, 10,000 CASMO-5M simulations were done to quantify the prior distribution of the calculated FP concentrations. MLO was also applied to account 


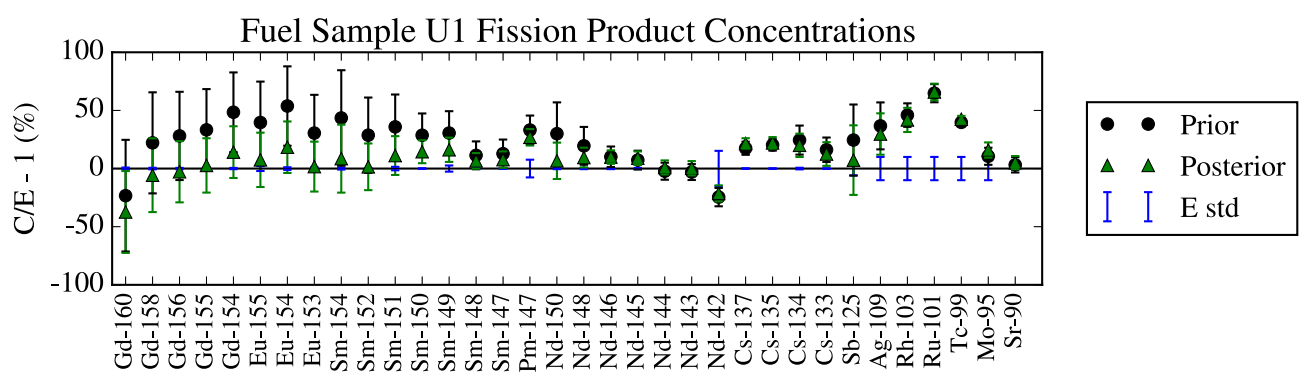

Figure 2. Prior and posterior biases and uncertainties of the simulated fission product concentrations of fuel sample U1.

for inconsistencies between the experiment and calculation and omitted sources of uncertainty, like the capture cross sections of the fission products. It created extra uncertainties for each integral datum that were added to $\mathbf{M}_{\mathbf{E}}$ in Eq. (3). With this prior distribution and the MLO extra uncertainties, we applied BFMC to update the GEF model parameters. We then re-sampled the posterior GEF model parameters and re-ran GEF to create posterior FY samples. Finally, we ran the CASMO-5M models of fuel samples $\mathrm{U} 1$ and $\mathrm{U} 2$ with the posterior FYs.

\section{Results}

\subsection{Posterior Fission Product Concentrations}

First, we examine how the posterior FYs performed in the CASMO-5M simulations of the LWR-PII fuel samples. We begin with fuel sample U1, or the training data used to adjust GEF's model parameters. The prior (before DA) and posterior (after DA) biases of the FP concentrations are presented in Fig. 2. The largest adjustments occurred to the isotopes of $\mathrm{Gd}, \mathrm{Eu}$, and $\mathrm{Sm}$. The average absolute value of the prior biases was $26 \%$ and the average relative standard deviation of the calculated FP concentrations was $21 \%$. After DA, the FP concentrations were recalculated with the posterior FYs. It reduced the average absolute value of the bias to $15 \%$ and the average relative standard deviation of the calculated values to $14 \%$.

Next, we examine how the DA performed when extrapolated to data that were not part of the training set, i.e. to fuel sample U2. This tests for over-fitting of GEF's model parameters to the U1 data. If they were indeed overfit, we expect worse biases for the posterior than for the prior. The average absolute value of the bias decreased from $19 \%$ in the prior to $8.7 \%$ in the posterior. The average relative standard deviation decreased from $19 \%$ to $13 \%$ as well. From these results, we conclude that the FYs were not over-fit to the U1 data because we have similar performance for U2's predicted FP concentrations.

\subsection{Posterior Fission Yields}

To analyze the posterior independent FYs, we calculated the relative difference between them and the FYs of ENDF/B-VIII.0. The relative differences are plotted for the FY means and for their standard deviations in Fig. 3 for
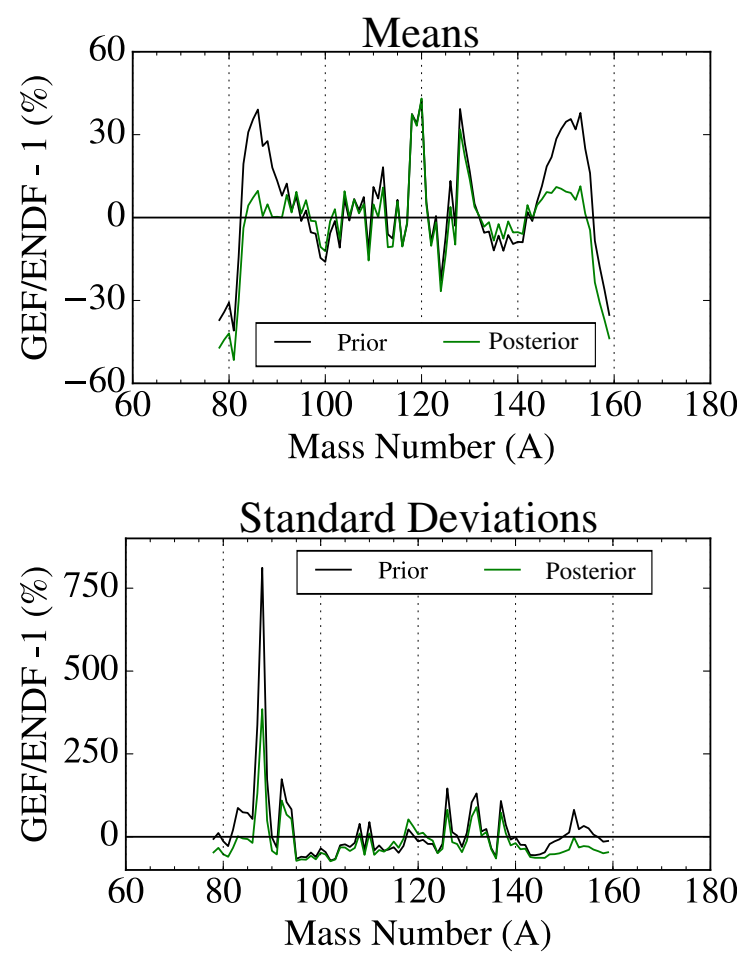

Figure 3. Relative differences between ENDF/B-VIII.0 and GEF of the means and standard deviations of the independent FY data for $\mathrm{Pu}-239$.

$\mathrm{Pu}-239$. In general, the relative differences of the means were between $\pm 40 \%$. On average, the absolute relative difference between the GEF and ENDF/B-VIII.0 decreased from $16 \%$ in the prior to $11 \%$ in the posterior. The biggest improvements of the means from prior to posterior occurred at $85<\mathrm{A}<95$ and $135<\mathrm{A}<150$. These improvements correspond to where experimental data were densest: $\sim 70 \%$ of the integral data had $\mathrm{A}>135$. Given the large concentration of evidence, the adjustments were the most significant there. When taken on average, the standard deviations of the prior GEF FYs were $16 \%$ larger than those from from ENDF/B-VIII.0. After the DA, the GEF FY uncertainties were $16 \%$ smaller. Similar to the mean values, the reductions in uncertainty are largest at 85 $<\mathrm{A}<95$ and $135<\mathrm{A}<150$. 

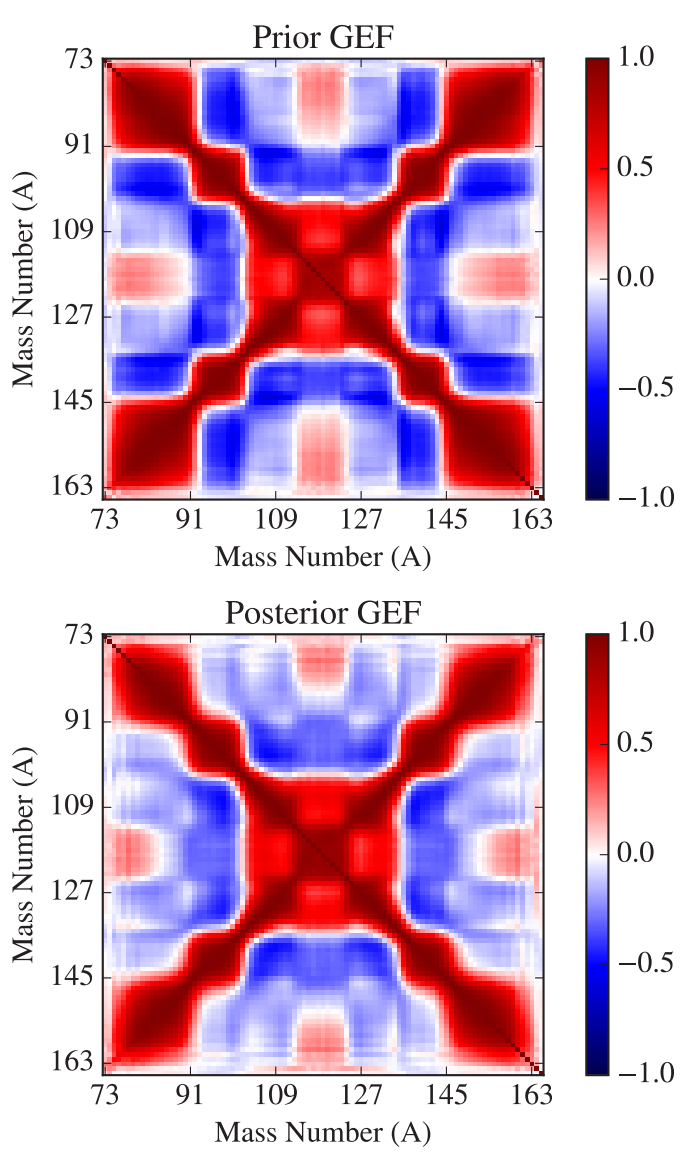

Figure 4. Correlations matrices of independent FYs of Pu-239.

Fig. 4 gives the prior and posterior correlation matrices for the independent FY data. The prior correlation matrix has large positive correlations (red) between FP pairs, which can be seen by following the diagonal from the lower-left to the upper-right. The strong correlations slightly off the main diagonal (upper-left to lower-right) come from FPs that are dominated by the same fission channel and are consequently highly correlated to the same model parameters. The matrix also has large degrees of anti-correlation (blue) between fission channels. This is related to the fact that these channels overlap on the FY spectrum and compete to create the FYs. DA reduced the degree of correlation between the FY data. The anticorrelation terms (in blue) were most affected, while the strong correlations, along the diagonal and between the two humps of the fission spectrum, were retained.

\section{Conclusions}

We demonstrated how PIE experiments can be used in DA to reduce the bias and uncertainty associated with predicted fission product concentrations in spent fuel. These improvements were made by adjusting the model parameters of the code GEF. The new model parameters changed the fission yields that were then used in the simulations of the spent fuel. Additionally, the DA produced posterior covariance matrices of the fission yields, which now have an additional experimental constraint.
DA improved the quality of the predicted concentrations of fission products in the studied spent fuel. Through the assimilation of the experimental data, the average bias of the simulated fission product concentrations decreased from $26 \%$ to $15 \%$. The average relative standard deviation decreased from $21 \%$ to $8.8 \%$. The posterior GEF fission yields agreed better with those in ENDF/B-VIII.0. For $\mathrm{Pu}-239$ thermal fission, the average relative difference from ENDF/B-VIII.0 was $16 \%$ for the prior and $11 \%$ for the posterior. For the standard deviations of the fission yields, the relative difference from ENDF/B-VIII.0 was $+16 \%$ prior and $-16 \%$ posterior to applying DA.

\section{References}

[1] O. Leray et al., Ann. Nucl. Energy 94 (2016)

[2] R. Mills, Nucl. Data Sheets 118 (2014)

[3] H. Sjöstrand et al., Nucl. Data Sheets 118 (2014)

[4] O. Leray et al., Prog. Nucl. Energy 101 (2017)

[5] G. Ilas, H. Liljenfeldt, Nucl, Eng, and Design 319 (2017)

[6] J.J. Herrero et al., EPJ Web Conf. 146 (2017)

[7] L. Fiorito et al., Ann. Nucl. Energy 88 (2016)

[8] K.H. Schmidt et al., Nucl. Data Sheets 131 (2016)

[9] C. Schmitt et al., Phys. Rev. C 98 (2018)

[10] K.H. Schmidt, B. Jurado, Rep. Progr. Phys. 81 (2018)

[11] D. Siefman, Ph.D. thesis, EPFL No. 7525 (2019)

[12] T. Frosio et al., EPJ Nuclear Sci. Technol. 4 (2018)

[13] A. Rizzo et al., EPJ Nuclear Sci. Technol. 4 (2018)

[14] G. Palmiotti et al., Nucl. Data Sheets 123 (2015)

[15] M. Salvatores et al., Nucl. Data Sheets 118 (2014)

[16] A. Hoefer et al., Ann. Nucl. Energy 77 (2015)

[17] E. Bauge et al., in Proceedings of ND2007 (2007)

[18] D. Rochman et al., Ann. Nucl. Energy 95 (2016)

[19] M. Chadwick et al., Nucl. Data Sheets 112 (2011)

[20] D. Siefman et al., Eur. Phys. J. Plus 133 (2018)

[21] D. Siefman et al., in Proceedings of MECC2017 (2017)

[22] H. Sjöstrand et al., in Proceedings of WONDER2018 (2018)

[23] G. Schnabel, in Proceedings of ME $C 2017$ (2017)

[24] P. Helgesson, Ph.D. thesis, Uppsala University, Applied Nuclear Physics (2018)

[25] M. Murphy et al., Ann. Nucl. Energy 33 (2006)

[26] P. Grimm et al., Prog. Nucl. Energy 101 (2017)

[27] P. Grimm et al., in Proceedings of PHYSOR2016 (2016)

[28] D. Brown et al., Nucl. Data Sheets 148 (2018)

[29] E. Alhassan et al., Prog. Nucl. Energy 88 (2016)

[30] D. Rochman et al., Eur. Phys. J. Plus 133 (2018)

[31] J. Rhodes et al., Tech. rep., Studsvik (2012)

[32] W. Wieselquist et al., Sci. and Technol. of Nucl. Install. (2013)

[33] M. Hursin et al., in Proceedings of PHYSOR2016 (2016)

[34] A. Aures et al., Ann. Nucl. Energy 101 (2017) 